\title{
OBSERVATIONAL CONSTRAINTS ON COSMOLOGICAL MODELS WITH CHAPLYGIN GAS AND QUADRATIC EQUATION OF STATE
}

\author{
G. S. Sharov ${ }^{1}$ \\ ${ }^{1}$ Tver state university \\ 170002, Sadovyj per. 35, Tver, Russid
}

\begin{abstract}
Observational manifestations of accelerated expansion of the universe, in particular, recent data for Type Ia supernovae, baryon acoustic oscillations, for the Hubble parameter $H(z)$ and cosmic microwave background constraints are described with different cosmological models. We compare the $\Lambda \mathrm{CDM}$, the models with generalized and modified Chaplygin gas and the model with quadratic equation of state. For these models we estimate optimal model parameters and their permissible errors with different approaches to calculation of sound horizon scale $r_{s}\left(z_{d}\right)$. Among the considered models the best value of $\chi^{2}$ is achieved for the model with quadratic equation of state, but it has 2 additional parameters in comparison with the $\Lambda \mathrm{CDM}$ and therefore is not favored by the Akaike information criterion.
\end{abstract}

\section{INTRODUCTION}

Observations [1, 2] of Type Ia supernovae demonstrated accelerated expansion of our universe. Further investigations of supernovae [3, 4], baryon acoustic oscillations [4 7], cosmic microwave background measurements [7-10], estimations [11 25] of the Hubble parameter $H(z)$ for different redshifts $z$ confirmed accelerated growth of the cosmological scale factor $a(t)$ at late stage of its evolution.

For Type Ia supernovae we can measure their redshifts $z$ and luminosity distances $D_{L}$, so these objects may be used as standard candles [1 4 4].

Baryon acoustic oscillations (BAO) are observed as a peak in the correlation function of the galaxy distribution at the comoving sound horizon scale $r_{s}\left(z_{d}\right)$ [5, 6], corresponding to the end of the drag era, when baryons became decoupled and acoustic waves propagation was ended. This effect has various observational manifestations 8 10, 16 33], in particular, one can estimate the Hubble parameter $H(z)$ for definite redshifts [16 25] (details are in Sect. III).

The mentioned recent observations of Type Ia supernovae, BAO and $H(z)$ essentially restrict possible cosmological theories and models. To satisfy these observations all models are to describe accelerated expansion of the universe with definite parameters $[8-10,34$ 36].

The standard Einstein gravity with $\Lambda=0$ predicts deceleration of the expanding universe: $a^{\prime \prime}(t)<0$. So to explain observed accelerated expansion, we are to modify this theory. The most simple (and most popular) modification is the $\Lambda \mathrm{CDM}$, including dark energy corresponding to $\Lambda \neq 0$ and cold dark matter in addition to deficient visible matter. This model with appropriate parameters [4, 8 -10] successfully describes practically all observational data, in Sect. III we apply this model to describe the updated recent observations of Type Ia supernovae, BAO effects and $H(z)$ estimates. In this paper we use the notation $\Lambda$ CDM for the model with an arbitrary spatial curvature $\Omega_{k}$.

One should note that there are some problems in the $\Lambda \mathrm{CDM}$ model, in particular, ambiguous nature of dark matter and dark energy, the problem of fine tuning for the observed value of $\Lambda$ and the coincidence problem for surprising proximity $\Omega_{\Lambda}$ and $\Omega_{m}$ nowadays [35, 36].

*Electronic address: german.sharov@mail.ru 
Therefore cosmologists suggested a lot of alternative models with different equations of state, scalar fields, with $f(R)$ Lagrangian, additional space dimensions and many others [35 39]. In this paper we consider in detail two models with nontrivial equations of state describing both dark matter and dark energy: the model with modified Chaplygin gas (MCG) [40-47] in Sect. IV] and the model with quadratic equation of state [48 53] in Sect. V.

\section{OBSERVATIONAL DATA}

In this paper we use the Union2.1 compilation [3] of Type Ia supernovae (SNe Ia) observational data. This table includes redshifts $z=z_{i}$ and distance moduli $\mu_{i}=\mu_{i}^{\text {obs }}$ with errors $\sigma_{i}$ for $N_{S N}=580$ supernovae. The distance modulus $\mu_{i}=\mu\left(D_{L}\right)=5 \log _{10}\left(D_{L} / 10 \mathrm{pc}\right)$ is logarithm of the luminosity distance $[1, \underline{34}, 35]$ :

$$
D_{L}(z)=\frac{c(1+z)}{H_{0}} S_{k}\left(H_{0} \int_{0}^{z} \frac{d \tilde{z}}{H(\tilde{z})}\right) .
$$

Here

$$
S_{k}(x)= \begin{cases}\sinh \left(x \sqrt{\Omega_{k}}\right) / \sqrt{\Omega_{k}}, & \Omega_{k}>0 \\ x, & \Omega_{k}=0 \\ \sin \left(x \sqrt{\left|\Omega_{k}\right|}\right) / \sqrt{\left|\Omega_{k}\right|}, & \Omega_{k}<0\end{cases}
$$

redshift $z$ and the Hubble parameter $H(z)$ are connected with the scale factor $a(t)$ :

$$
a(t)=\frac{a_{0}}{1+z}, \quad H(z)=\frac{\dot{a}(t)}{a(t)}
$$

$k$ is the sign of curvature, $\Omega_{k}=-k /\left(a_{0}^{2} H_{0}^{2}\right)$ is its present time fraction, $a_{0} \equiv a\left(t_{0}\right)$ and $H_{0} \equiv H\left(t_{0}\right)$ are the current values of $a$ and $H$.

For any cosmological model we fix its model parameters $p_{1}, p_{2}, \ldots$, calculate dependence $a(t)$, the integral (11) and this model predicts theoretical values $D_{L}^{t h}$ for luminosity distance (1) (for given $z$ ), or $\mu^{\text {th }}$ for modulus. To compare these theoretical values with the observational data $z_{i}$ and $\mu_{i}^{o b s}$ from the table [3] we use the function

$$
\chi_{S N}^{2}\left(p_{1}, p_{2}, \ldots\right)=\min _{H_{0}} \sum_{i, j=1}^{N_{S N}} \Delta \mu_{i}\left(C_{S N}^{-1}\right)_{i j} \Delta \mu_{j}, \quad \Delta \mu_{i}=\mu^{t h}\left(z_{i}, H_{0}, p_{1}, \ldots\right)-\mu_{i}^{o b s} .
$$

Here $C_{S N}$ is the SN-by-SN covariance matrix [3], representing systematic errors.

In the sum (11) marginalization over the Hubble constant $H_{0}$ is assumed, because we have to take into account model dependence of the moduli $\mu_{i}^{o b s}$. Unlike observed apparent magnitudes $m_{i}^{o b s}$ the values $\mu_{i}^{o b s}$ in Ref. [3] are estimated as

$$
\mu^{o b s}=m^{o b s}(z)-M+\alpha x_{1}-\beta c+\delta P .
$$

This formula includes the SN Ia absolute magnitude $M$ and corrections connected with deviations from mean values of lightcurve shape $\left(x_{1}\right)$, SN Ia color $(c)$ and mass of a host galaxy (the factor $P)$. The parameters $M, \alpha, \beta$ and $\delta$ are considered in Ref. [3] as nuisance parameters, they are fitted simultaneously with the cosmological parameters in the flat $\Lambda$ CDM model.

Thus we have a model dependent additive term in Eq. (44) for the Union2.1 values $\mu^{\text {obs }}$ [3] with concealed dependence on the Hubble constant $H_{0}$ and other model parameters. In particular, one 
can find only that the best fit value for the absolute magnitude $M=-19.321 \pm 0.03$ is obtained in Ref. [3] for $h=0.7$, where $h=H_{0} / 100 \mathrm{~km} \mathrm{~s}^{-1} \mathrm{Mpc}^{-1}$.

To suppress this dependence many authors [54 57] suppose that values $\mu_{i}^{\text {obs }}$ from any SN Ia survey have a systematic error depending on $H_{0}$ and marginalize the sum (1) over the Hubble constant $H_{0}$. They use the fact, that for the most popular models theoretical value of the luminosity distance (10) depends on $H_{0}$ as $D_{L}^{t h} \sim H_{0}^{-1}$, so the distance modulus $\mu^{t h}$ has the additive term $-5 \log _{10} H_{0}$. In Ref. [54] this term is separated as $\mu_{0}$ in the form

$$
\mu\left(D_{L}\right)=5 \log _{10} \frac{H_{0} D_{L}}{c}+\mu_{0}, \quad \mu_{0}=42.384-5 \log _{10} h ;
$$

if we denote $d_{i}=\Delta \mu_{i}-\mu_{0}, \mathbf{d}=\left(d_{1}, \ldots, d_{S N}\right)$, the minimum of the sum (3) over $H_{0}$ (or over $\left.\mu_{0}\right)$ will take the form

$$
\chi_{S N}^{2}\left(p_{1}, \ldots\right)=\mathbf{d} C_{S N}^{-1} \mathbf{d}^{T}-\frac{B^{2}}{C}, \quad B=\sum_{i, j=1}^{N_{S N}} d_{i}\left(C_{S N}^{-1}\right)_{i j}, \quad C=\sum_{i, j=1}^{N_{S N}}\left(C_{S N}^{-1}\right)_{i j} .
$$

In this paper for all models we use the marginalized function (5) $\chi_{S N}^{2}$ to describe the supernovae Ia data [3].

This approach with separation of the Hubble constant $H_{0}$ among other model parameters can not be applied to $H(z)$ and BAO observational data, because observed values have different dependence on $H_{0}$.

To describe the BAO data we calculate the distance [5, 8- 10]

$$
D_{V}(z)=\left[\frac{c z D_{L}^{2}(z)}{(1+z)^{2} H(z)}\right]^{1 / 3}
$$

and two measured values

$$
d_{z}(z)=\frac{r_{s}\left(z_{d}\right)}{D_{V}(z)}, \quad A(z)=\frac{H_{0} \sqrt{\Omega_{m}}}{c z} D_{V}(z)
$$

which are usually considered as observational manifestations of baryon acoustic oscillations [5, 8]. Here $\Omega_{m}=\frac{8}{3} \pi G \rho\left(t_{0}\right) / H_{0}^{2}$ is the present time fraction of matter with density $\rho$. The value $r_{s}\left(z_{d}\right)$ in Eq. (77) is sound horizon size at the end of the drag era $z_{d}$ :

$$
r_{s}\left(z_{d}\right)=\int_{z_{d}}^{\infty} \frac{c_{s}(z)}{H(z)} d z
$$

To estimate this important parameter different authors [17 33] used theoretical or statistical approaches and suggested different fitting formulas for $r_{s}$. In table \ the following recent estimations of $r_{s}\left(z_{d}\right) \equiv r_{d}$ and $r_{d} h$ are shown:

\begin{tabular}{||c||c|c|c|c|c|c|c|c|c|c|c||}
\hline Refs & {$[25]$} & {$[9,24]$} & {$[10]$} & {$[32]$} & {$[33]$} & {$[22]$} & {$[29]$} & {$[18]$} & {$[21]$} & {$[27,28,31]$} & {$[26]$} \\
\hline$r_{s}\left(z_{d}\right)$ & 147.4 & 147.49 & 147.6 & 148.6 & 148.69 & 149.28 & 152.40 & 152.76 & 153.19 & 153.2 & 153.5 \\
\hline$r_{d} \cdot h$ & 98.79 & 99.26 & 100.09 & 99.78 & 99.62 & 104.49 & 105.15 & 106.93 & 107.23 & 103.6 & 104.69 \\
\hline
\end{tabular}

TABLE I: Recent estimations of $r_{s}\left(z_{d}\right)$ and $r_{s}\left(z_{d}\right) \cdot h(\mathrm{Mpc})$.

In the most of cited papers in table \ the values $r_{s}\left(z_{d}\right)$ were considered as fiducial ones for calculating $D_{V}(z), H(z)$ and other parameters. So these results sufficiently depend on $r_{s}\left(z_{d}\right)$, in 
particular, estimations of $H(z)$ from the BAO data [16 25] are defined with the factor $r_{d}^{f i d} / r_{d}$, the corresponding factor $r_{d} / r_{d}^{f i d}$ takes place for calculated values $D_{V}(z)$ or $D_{A}(z)=D_{L}(z) /(1+z)^{2}$.

In this paper we use two different approaches to calculate the sound horizon scale $r_{s}\left(z_{d}\right)$. But previously one should mention the simplest method, applied in Ref. [39], where the arithmetic average of the $r_{d}$ values in table \ (with their multiplicity)

$$
r_{s}\left(z_{d}\right)=150.69 \pm 2.45 \mathrm{Mpc}
$$

was used as the basic value. This value is independent on $H_{0}$, hence the observational parameter $d_{z}(z)$ in Eq. (7) appears to be Hubble dependent (though the formula (8) predicts $r_{d} \sim H_{0}^{-1}$ and Hubble free $d_{z}$ ). One may conclude, that $h$ dependence of $d_{z}$ is the drawback of this approach, so in this paper we consider the fixed value $r_{d}$ (9) only in section III to emphasize advantages of other methods.

More appropriate procedures to calculate $r_{s}\left(z_{d}\right)$ include different fitting formulae [6, 7, 22, 57]. In this paper we use the numerically calibrated approximation from Ref. [7]

$$
r_{s}\left(z_{d}\right)=\frac{55.154 \exp \left[72.3\left(\Omega_{\nu} h^{2}+0.0006\right)^{2}\right]}{\left(\Omega_{m} h^{2}\right)^{0.25351}\left(\Omega_{b} h^{2}\right)^{0.12807}} \mathrm{Mpc}
$$

as the basic formula. The resulting $h$ dependence in Eq. (10) (for a reasonable neutrino contribution with $\left.\sum m_{\nu} \leq 0.23 \mathrm{eV}[10]\right)$ is $r_{d} \sim h^{-0.7632}$, it is more close to the true variant $r_{d} \sim h^{-1}$. The dependence on $\Omega_{m}$ in Eq. (10) is well fitted for $\Lambda$ CDM-like models, however for the models with Chaplygin gas and with quadratic EoS, considered below, $\Omega_{m}$ is not a basic model parameter. The value $\Omega_{m}$ in these models should be estimated in a special way, so an additional uncertainty appears in this approach.

Thus, an alternative simple fitting formula

$$
r_{s}\left(z_{d}\right)=\frac{\left(r_{d} \cdot h\right)_{f i d}}{h}, \quad\left(r_{d} \cdot h\right)_{f i d}=104.57 \mathrm{Mpc} .
$$

with true $h$ dependence may be suggested. Here the value $\left(r_{d} \cdot h\right)_{f i d}=104.57 \pm 1.44 \mathrm{Mpc}$ was chosen as the best fit for the $\Lambda \mathrm{CDM}$ model. This procedure is described in the next section and illustrated in figure 1 .

The parameter $\left(r_{d} \cdot h\right)_{f i d}$ for the expression (11) plays the same role as the baryonic fraction $\Omega_{b}$ for the formula (10), in both cases we do not consider $\Omega_{b}$ and $r_{d} h$ as free model parameters for all models, but fix their optimal (fiducial) values after description of the simplest $\Lambda$ CDM model. The best $\Lambda \mathrm{CDM}$ fit for $\Omega_{b}$ in Eq. (10) (see figure 1) is

$$
\Omega_{b}=0.044 \pm 0.004 \text {. }
$$

To take into account all available BAO data [16 33] for parameters (7), we consider in this paper $N_{B A O}=17$ data points for $d_{z}(z)$ (10 additional points in comparison with the table in our paper [39]) and 7 data points for $A(z)$ presented in the table II]

Measurements of $d_{z}(z)$ and $A(z)$ from Refs. [26, 29] in table II are not independent. So the $\chi^{2}$ function for the values (7) is

$$
\chi_{B A O}^{2}\left(p_{1}, p_{2}, \ldots\right)=(\Delta d)^{T} C_{d}^{-1} \Delta d+(\Delta A)^{T} C_{A}^{-1} \Delta A, \quad \Delta d=d_{z}\left(z_{i}\right)-d_{z}^{\text {th }} .
$$

The elements of covariance matrices $C_{d}^{-1}=\left\|c_{i j}^{d}\right\|$ and $C_{A}^{-1}=\left\|c_{i j}^{A}\right\|$ in Eq. (13) are [8, 26, 29]:

$$
\begin{array}{llll}
c_{33}^{d}=30124, & c_{38}^{d}=-17227, & c_{88}^{d}=86977, & \\
c_{1111}^{d}=24532.1, & c_{1114}^{d}=-25137.7, & c_{1115}^{d}=12099.1, & c_{1414}^{d}=134598.4, \\
c_{1415}^{d}=-64783.9, & c_{1515}^{d}=128837.6 ; & c_{1111}^{A}=1040.3, & c_{1114}^{A}=-807.5, \\
c_{1115}^{A}=336.8, & c_{1414}^{A}=3720.3, & c_{1415}^{A}=-1551.9, & c_{1515}^{A}=2914.9 .
\end{array}
$$




\begin{tabular}{||l|l|l|l|l|c|l||}
\hline$z$ & $d_{z}(z)$ & $\sigma_{d}$ & $A(z)$ & $\sigma_{A}$ & Refs & Survey \\
\hline 0.106 & 0.336 & 0.015 & 0.526 & 0.028 & {$[\underline{8}, 28]$} & 6dFGS \\
\hline 0.15 & 0.2232 & 0.0084 & - & - & {$[33]$} & SDSS DR7 \\
\hline 0.20 & 0.1905 & 0.0061 & 0.488 & 0.016 & {$[26,29]$} & SDSS DR7 \\
\hline 0.275 & 0.1390 & 0.0037 & - & - & {$[26]$} & SDSS DR7 \\
\hline 0.278 & 0.1394 & 0.0049 & - & - & {$[27]$} & SDSS DR7 \\
\hline 0.314 & 0.1239 & 0.0033 & - & - & {$[29]$} & SDSS LRG \\
\hline 0.32 & 0.1181 & 0.0026 & - & - & {$[22]$} & BOSS DR11 \\
\hline 0.35 & 0.1097 & 0.0036 & 0.484 & 0.016 & {$[26,29]$} & SDSS DR7 \\
\hline 0.35 & 0.1126 & 0.0022 & - & - & {$[30]$} & SDSS DR7 \\
\hline 0.35 & 0.1161 & 0.0146 & - & - & {$[19]$} & SDSS DR7 \\
\hline 0.44 & 0.0916 & 0.0071 & 0.474 & 0.034 & {$[29]$} & WiggleZ \\
\hline 0.57 & 0.0739 & 0.0043 & 0.436 & 0.017 & {$[20]$} & SDSS DR9 \\
\hline 0.57 & 0.0726 & 0.0014 & - & - & {$[22]$} & SDSS DR11 \\
\hline 0.60 & 0.0726 & 0.0034 & 0.442 & 0.020 & {$[29]$} & WiggleZ \\
\hline 0.73 & 0.0592 & 0.0032 & 0.424 & 0.021 & {$[29]$} & WiggleZ \\
\hline 2.34 & 0.0320 & 0.0021 & - & - & {$[25]$} & BOSS DR11 \\
\hline 2.36 & 0.0329 & 0.0017 & - & - & {$[24]$} & BOSS DR11 \\
\hline
\end{tabular}

TABLE II: Values of $d_{z}(z)=r_{s}\left(z_{d}\right) / D_{V}(z)$ and $A(z)$ (17) with errors and references

Here $c_{i j}=c_{j i}$, the remaining matrix elements are $c_{i i}=1 / \sigma_{i}^{2}, c_{i j}=0, i \neq j$.

In the values $\sigma_{d}$ in table $\amalg$ we took into account correlation between estimations of $d_{z}(z)$ and $H(z)$ (table III) for $z=0.35,0.57,2.34,2.36$ in Refs. [19 21, 24, 25].

Measurements of the Hubble parameter $H(z)$ for different redshifts $z$ with 38 data points [11 25] are presented in table III. These values $H(z)$ were calculated with two methods: 1$)$ differential age approach in Refs. [11 15] with evaluation of the age difference $d t$ for galaxies with close redshifts $d z$ and the formula

$$
H(z)=\frac{1}{a(t)} \frac{d a}{d t}=-\frac{1}{1+z} \frac{d z}{d t}
$$

2) measurement [16 25] of the BAO peak in the correlation function in line-of-sight directions at a redshift separation $\Delta z=r_{s}\left(z_{d}\right) H(z) / c$.

For the latter method estimations [16 25] of $H(z)$ essentially depend on a fiducial value $r_{d}^{f i d}$ and have the factor $r_{d}^{f i d} / r_{d}$, as was mentioned above. In particular, the result in Ref. [25] is

$$
H(z=2.34)=(222 \pm 7) \frac{\mathrm{km}}{\mathrm{s} \cdot \mathrm{Mpc}} \cdot \frac{147.4 \mathrm{Mpc}}{r_{s}\left(z_{d}\right)} .
$$

In table III this factor is taken into account only for the errors $\sigma_{H}$ from the papers [16, 1820, 24, 25], where fiducial values $r_{d}^{f i d}$ essentially differ from the average (9). The estimations for $H(z)$ in table III are the same as in the correspondent sources.

To compare the $H(z)$ data in table III with $N_{H}=38$ data points with model predictions we use the $\chi^{2}$ function

$$
\chi_{H}^{2}\left(p_{1}, p_{2}, \ldots\right)=\sum_{i=1}^{N_{H}} \frac{\left[H_{i}-H^{t h}\left(z_{i}, p_{1}, p_{2}, \ldots\right)\right]^{2}}{\sigma_{H, i}^{2}},
$$

similar to the function (3) for the SN Ia observational data from Ref. [3]. 


\begin{tabular}{||l|l|l|l||l|l|l|l||}
\hline$z$ & $H(z)$ & $\sigma_{H}$ & Refs & $z$ & $H(z)$ & $\sigma_{H}$ & Refs \\
\hline 0.070 & 69 & 19.6 & {$[14]$} & 0.570 & 96.8 & 3.4 & {$[22]$} \\
\hline 0.090 & 69 & 12 & {$[11]$} & 0.593 & 104 & 13 & {$[13]$} \\
\hline 0.120 & 68.6 & 26.2 & {$[14]$} & 0.600 & 87.9 & 6.1 & {$[17]$} \\
\hline 0.170 & 83 & 8 & {$[11]$} & 0.680 & 92 & 8 & {$[13]$} \\
\hline 0.179 & 75 & 4 & {$[13]$} & 0.730 & 97.3 & 7.0 & {$[17]$} \\
\hline 0.199 & 75 & 5 & {$[13]$} & 0.781 & 105 & 12 & {$[13]$} \\
\hline 0.200 & 72.9 & 29.6 & {$[14]$} & 0.875 & 125 & 17 & {$[13]$} \\
\hline 0.240 & 79.69 & 2.99 & {$[16]$} & 0.880 & 90 & 40 & {$[12]$} \\
\hline 0.270 & 77 & 14 & {$[11]$} & 0.900 & 117 & 23 & {$[11]$} \\
\hline 0.280 & 88.8 & 36.6 & {$[14]$} & 1.037 & 154 & 20 & {$[13]$} \\
\hline 0.300 & 81.7 & 6.22 & {$[23]$} & 1.300 & 168 & 17 & {$[11]$} \\
\hline 0.340 & 83.8 & 3.66 & {$[16]$} & 1.363 & 160 & 33.6 & {$[15]$} \\
\hline 0.350 & 82.7 & 9.1 & {$[19]$} & 1.430 & 177 & 18 & {$[11]$} \\
\hline 0.352 & 83 & 14 & {$[13]$} & 1.530 & 140 & 14 & {$[11]$} \\
\hline 0.400 & 95 & 17 & {$[11]$} & 1.750 & 202 & 40 & {$[11]$} \\
\hline 0.430 & 86.45 & 3.97 & {$[16]$} & 1.965 & 186.5 & 50.4 & {$[15]$} \\
\hline 0.440 & 82.6 & 7.8 & {$[17]$} & 2.300 & 224 & 8.6 & {$[18]$} \\
\hline 0.480 & 97 & 62 & {$[12]$} & 2.340 & 222 & 8.5 & {$[25]$} \\
\hline 0.570 & 87.6 & 7.8 & {$[20]$} & 2.360 & 226 & 9.3 & {$[24]$} \\
\hline
\end{tabular}

TABLE III: Values of the Hubble parameter $H(z)$ with errors $\sigma_{H}$ from Refs. 11 25]

We mentioned above that the observed values in tables II, III have different dependence on $h=H_{0} / 100 \mathrm{~km} \mathrm{~s}^{-1} \mathrm{Mpc}^{-1}$. In particular, if we use the fitting formula (10) for $r_{s}\left(z_{d}\right)$, the parameter $d_{z}$ (77) has rather weak $h$ dependence $\left(d_{z} \sim h^{0.2368}\right.$, because $\left.D_{V} \sim h^{-1}\right)$; for the formula (11) $d_{z}$ is Hubble free in accordance with Eq. (7). The value $A(z)$ is also Hubble free, but it depends on $\Omega_{m}$. The values $H(z)$ in table III are naturally proportional to $H_{0}$. So estimations in tables II, III may be model dependent, but we can assume that different authors use different methods and produce possible systematic errors for $d_{z}(z)$ and $H(z)$ in different directions. One can suppose mean systematic errors to be close to zero.

On the other hand, if any form of marginalization over $H_{0}$ for BAO and $H(z)$ data is made 55 57], the obtained results will have an additional error, because a model can successfully describe all SN Ia, BAO and $H(z)$ data, but with 3 essentially different intrinsic values of $H_{0}$. Under these arguments we make the marginalization procedure (5) over $H_{0}$ only for SN Ia data [3], but not for $\mathrm{BAO}$ and $H(z)$ data from tables $\amalg$, III.

\section{III. $\Lambda$ CDM MODEL}

In the $\Lambda \mathrm{CDM}$ and other models in this paper the Einstein equations

$$
G_{\nu}^{\mu}=8 \pi G T_{\nu}^{\mu}-\Lambda \delta_{\nu}^{\mu}
$$

describe dynamics of the universe. Here $G_{\nu}^{\mu}=R_{\nu}^{\mu}-\frac{1}{2} R \delta_{\nu}^{\mu}, T_{\nu}^{\mu}=\operatorname{diag}(-\rho, p, p, p)$.

In the $\Lambda \mathrm{CDM}$ model baryonic and dark matter may be described as one component of dust-like matter with density $\rho=\rho_{c}=\rho_{b}+\rho_{d m}$, so we suppose $p=0$ in $T_{\nu}^{\mu}$. In models with Chaplygin gas (Sect. IV) and with quadratic equation of state (Sect. V) we suppose that an additional component of matter describes both dark matter and dark energy and gives some contribution $\rho_{g}$ in the total 
density:

$$
\rho=\rho_{c}+\rho_{g}+\rho_{r}
$$

The fraction of relativistic matter (radiation and neutrinos) is close to zero for observable values $z \leq 2.36$, so below we suppose $\rho_{r}=0$.

For the Robertson-Walker metric with the curvature sign $k$

$$
d s^{2}=-d t^{2}+a^{2}(t)\left[\left(1-k r^{2}\right)^{-1} d r^{2}+r^{2} d \Omega\right]
$$

the Einstein equations (15) are reduced to the system

$$
\begin{aligned}
3 \frac{\dot{a}^{2}+k}{a^{2}} & =8 \pi G \rho+\Lambda, \\
\dot{\rho} & =-3 \frac{\dot{a}}{a}(\rho+p) .
\end{aligned}
$$

Eq. (19) results from the continuity condition $T_{\nu ; \mu}^{\mu}=0$, the dot denotes the time derivative, here and below the speed of light $c=1$.

For the $\Lambda$ CDM with dust-like matter $(p=0)$ we use the solution of Eq. (19) $\rho / \rho_{0}=\left(a / a_{0}\right)^{-3}$ and rewrite Eq. (18) in the form

$$
\frac{\dot{a}^{2}}{a^{2} H_{0}^{2}}=\frac{H^{2}}{H_{0}^{2}}=\Omega_{m}\left(\frac{a}{a_{0}}\right)^{-3}+\Omega_{\Lambda}+\Omega_{k}\left(\frac{a}{a_{0}}\right)^{-2} .
$$

Here the present time fractions of matter, dark energy ( $\Lambda$ term) and curvature

$$
\Omega_{m}=\frac{8 \pi G \rho\left(t_{0}\right)}{3 H_{0}^{2}}, \quad \Omega_{\Lambda}=\frac{\Lambda}{3 H_{0}^{2}}, \quad \Omega_{k}=-\frac{k}{a_{0}^{2} H_{0}^{2}}
$$

are connected by the equality

$$
\Omega_{m}+\Omega_{\Lambda}+\Omega_{k}=1
$$

resulting from Eq. (20) if we fix $t=t_{0}$.

If we introduce dimensionless time $\tau$ and logarithm of the scale factor [38]

$$
\tau=H_{0} t, \quad \mathcal{A}=\log \frac{a}{a_{0}} .
$$

equation (201) will take the form $\frac{d \mathcal{A}}{d \tau}=\sqrt{\Omega_{m} e^{-3 \mathcal{A}}+\Omega_{\Lambda}+\Omega_{k} e^{-2 \mathcal{A}}}$, more convenient for numerical solving with the initial condition at the present time $\left.\mathcal{A}\right|_{\tau=1}=0$ equivalent to $a\left(t_{0}\right)=a_{0}$. Here and below the present time $t=t_{0}$ corresponds to $\tau=1$.

If we fix all model parameters, we can solve numerically the Cauchy problem for Eq. (20) and calculate the values $a(t) / a_{0}, H(z), D_{L}(z)$ (11), $d_{z}(z)$ and $A(z)$ (7). To compare them with the observational data from Ref. [3] and tables [I] [II] we use the $\chi^{2}$ functions (3), (13) and (14) and (under assumption about their Gaussian nature) the correspondent summarized function

$$
\chi_{\Sigma}^{2}=\chi_{S N}^{2}+\chi_{H}^{2}+\chi_{B A O}^{2} .
$$

When we apply the $\Lambda$ CDM model for describing the observational data from Sect. III (for $z \leq 2.36$ ), we use three free model parameters $H_{0}, \Omega_{m}$ and $\Omega_{\Lambda}$ (or $\Omega_{k}$ instead of $\Omega_{\Lambda}$ ) and the additional parameter

$$
\Omega_{b}=\frac{\rho_{b}\left(t_{0}\right)}{\rho_{c r}}=\frac{8 \pi G \rho_{b}\left(t_{0}\right)}{3 H_{0}^{2}}
$$


if we use the fitting formula (10) for $r_{s}\left(z_{d}\right)$. For the formula (11) the baryonic fraction $\Omega_{b}$ is not a model parameter, we mentioned above that the value $r_{d} \cdot h$ plays the role of an additional parameter in the case (11). In both approaches we test the $\Lambda$ CDM model and estimate the best $\Lambda$ CDM fit correspondingly for $\Omega_{b}$ and $r_{d} \cdot h$.

The results are presented in figure 1, where dependence of the best (minimal) value of the function (24) $\min \chi_{\Sigma}^{2}=\min _{H_{0}, \Omega_{m}, \Omega_{\Lambda}} \chi_{\Sigma}^{2}$ on $\Omega_{b}$ is shown in the top-left panel for the case (10). Here and for all models we assume $\sum m_{\nu}=0.06 \mathrm{eV}$ [7, 9]. One should note that the formula (10) is insensitive to a neutrino contribution in the range $\sum m_{\nu} \leq 0.23 \mathrm{eV}[10]$.

For the variant with Eq. (11) the similar dependence of $\min \chi_{\Sigma}^{2}$ on $r_{d} \cdot h$ is in the top-right panel. Here and below we draw these graphs as solid red lines for the fitting formula (10) and as dashed blue lines for the variant (11).
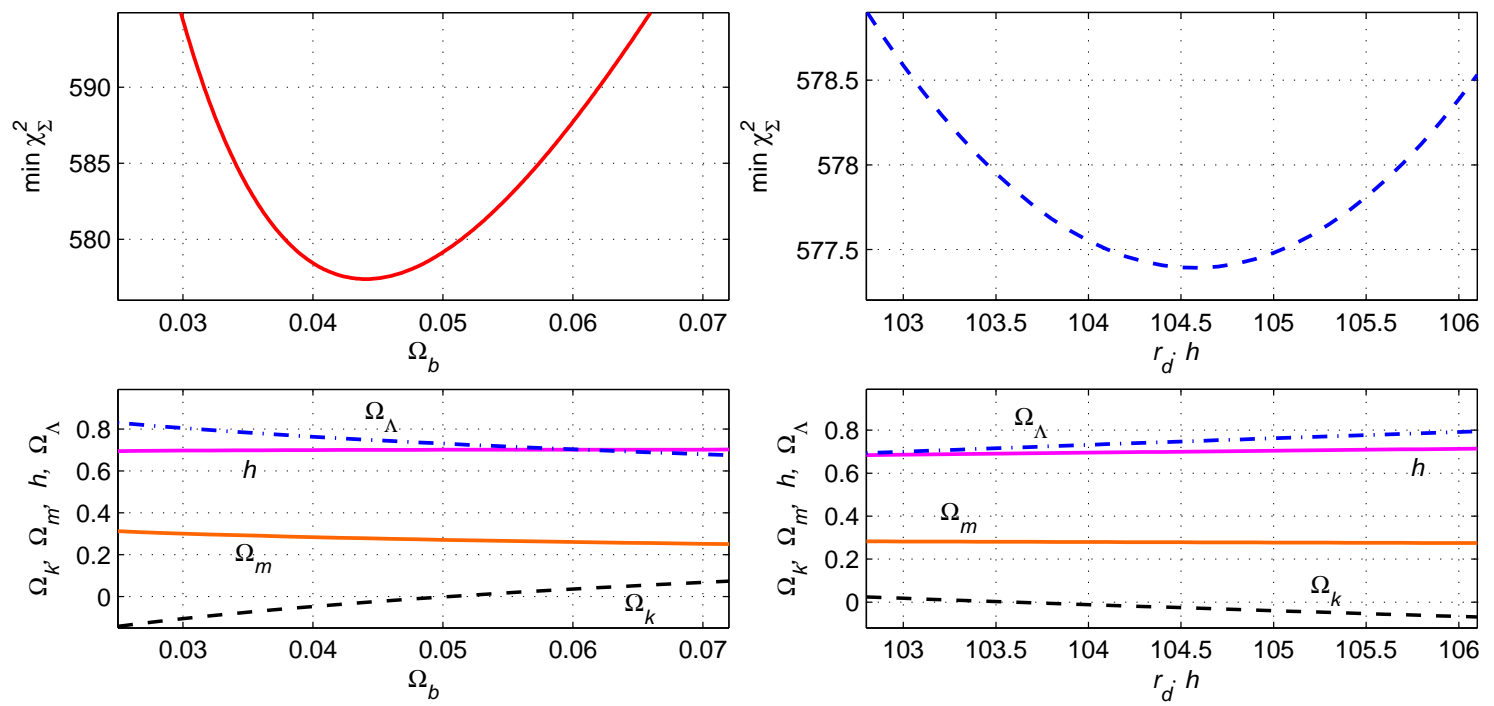

FIG. 1: The $\Lambda$ CDM model: dependence of the best fit $\min _{H_{0}, \Omega_{m}, \Omega_{\Lambda}} \chi_{\Sigma}^{2}$ on $\Omega_{b}$ for the fitting formula (10) (the left panels) and on $r_{d} \cdot h$ for Eq. (11) (the right panels). In the bottom panels we show the correspondent dependencies for parameters $H_{0}, \Omega_{m}, \Omega_{\Lambda}, \Omega_{k}$ of the $\chi_{\Sigma}^{2}$ minimum point.

The bottom panels of figure 1 illustrate how coordinates $h, \Omega_{m}, \Omega_{\Lambda}$ and $\Omega_{k}=1-\Omega_{m}-\Omega_{\Lambda}$ of the minimum point for $\min _{H_{0}, \Omega_{m}, \Omega_{\Lambda}} \chi_{\Sigma}^{2}$ depend on the correspondent parameters $\Omega_{b}$ and $r_{d} \cdot h$.

One can see that for the formula (10) dependence of $\min \chi_{\Sigma}^{2}$ on $\Omega_{b}$ is rather sharp: we have the distinct minimum at the value (12) $\Omega_{b}=0.044$. Below we use this value as the fiducial one for all models. The correspondent dependence in the top-right panel is more smooth, however it results in the optimal (fiducial) value $r_{d} \cdot h=104.57 \pm 1.44 \mathrm{Mpc}$ in the formula (11).

If we fix these parameters as described above, we can test the $\Lambda$ CDM model for different values of the remaining 3 parameters: $H_{0}, \Omega_{m}$ and $\Omega_{\Lambda}$. The results of calculations are presented in tables IV, V] VI and in figure 2. In the top-left panel of the figure we see how minimum of the function (24) $\min \chi_{\Sigma}^{2}=\min _{\Omega_{m}, \Omega_{\Lambda}} \chi_{\Sigma}^{2}\left(H_{0}\right)$ depend on the Hubble constant $H_{0}$ : red solid lines in the top panels describe the model with the formula (10), blue dashed lines correspond to the variant (11). For the sake of comparison we present here graphs for the fixed value $r_{s}\left(z_{d}\right)$ (9) as green lines with dots. These minima are calculated for each fixed value $H_{0}$.

One may see that the function $\min \chi_{\Sigma}^{2}\left(H_{0}\right)$ for the fitting formula (10) (the red line) has the maximal spread and achieves its minimum $\min \chi_{\Sigma}^{2} \simeq 577.39$ at

$$
H_{0}=70.07 \pm 1.82 \mathrm{~km}(\mathrm{~s} \mathrm{Mpc})^{-1}
$$



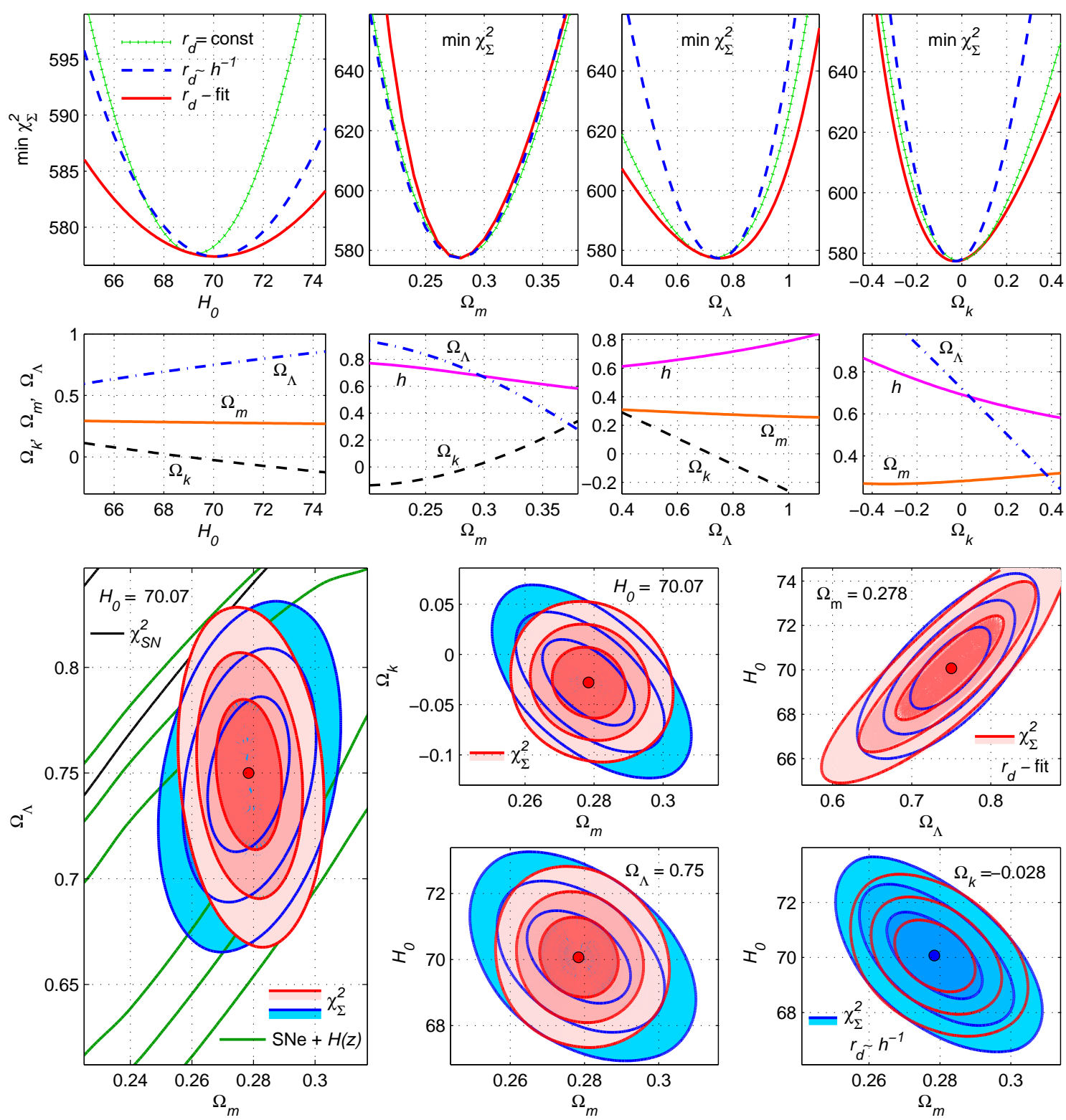

FIG. 2: For the $\Lambda \mathrm{CDM}$ in the top panels we present how $\min \chi_{\Sigma}^{2}$ depends on $H_{0}, \Omega_{m}, \Omega_{\Lambda}$ and $\Omega_{k}$ with different fitting formulae for $r_{d}$ : for Eq. (10) with $\Omega_{b}=0.044$ (red solid lines), for Eq. (11) (blue dashed lines), for Eq. (9) (green lines with dots). The correspondent dependencies for parameters of $\chi_{\Sigma}^{2}$ minimum point for Eq. (10) are shown in the 2-nd row. In other panels $1 \sigma, 2 \sigma$ and $3 \sigma$ level lines are drawn for $\chi_{\Sigma}^{2}$ as red filled contours for Eq. (10) and blue filled contours for Eq. (11) and also as black lines for $\chi_{S N}^{2}$ and green lines for $\chi_{S N}^{2}+\chi_{H}^{2}$; optimal values of the third parameter are fixed and shown.

for the following values of other parameters: $\Omega_{m} \simeq 0.278, \Omega_{\Lambda} \simeq 0.75$ (presented in table IV). It is interesting to see in table IV and in figure 2 that for the fitting formula (11) (blue dashed lines) the minimum $\min \chi_{\Sigma}^{2}$ and the optimal values of all parameters are the same, there is some difference only in $1 \sigma$ errors. To estimate $1 \sigma$ errors in the equality (26) we use the one-dimensional likelihood function $\mathcal{L}_{\Sigma}\left(H_{0}\right) \sim \exp \left(-\chi_{\Sigma}^{2} / 2\right)$ corresponding to $\min _{\Omega_{m}, \Omega_{\Lambda}} \chi_{\Sigma}^{2}\left(H_{0}\right)$.

Optimal values and errors for other model parameters $\Omega_{m}, \Omega_{\Lambda}, \Omega_{k}$ are calculated similarly, they are tabulated below in table IV in comparison with other estimates and also in the next section in 
tables $\mathrm{V}$ and VI correspondingly for the expressions (10) and (11).

Estimations of $\Omega_{m}$ and $\Omega_{\Lambda}$ in tables IV, V] VI are connected with graphs in the next two panels in the top line of figure 2, which present how minima of $\chi_{\Sigma}^{2}$ depend on $\Omega_{m}$ and on $\Omega_{\Lambda}$. In particular, the solid red line in the second top panel describes $\min \chi_{\Sigma}^{2}\left(\Omega_{m}\right)=\min _{H_{0}, \Omega_{\Lambda}} \chi_{\Sigma}^{2}$; the corresponding likelihood function $\mathcal{L}_{\Sigma}\left(\Omega_{m}\right) \sim \exp \left(-\chi_{\Sigma}^{2} / 2\right)$ determines the $1 \sigma$ error $\Delta \Omega_{m} \simeq 0.008$ in tables IV, V. This panel demonstrates that dependencies of $\min \chi_{\Sigma}^{2}$ on $\Omega_{m}$ for different variants of $r_{s}\left(z_{d}\right)$ are rather close and have rather sharp form. It is connected with the contribution in $\chi_{B A O}^{2}$ from the value $A(z)$ (7), because $A(z)$ is proportional to $\sqrt{\Omega_{m}}$ and $\chi_{B A O}^{2}$ is very sensitive to $\Omega_{m}$ values. For the red line in this panel we have the additional dependence on $\Omega_{m}$ in the formula (10). In the third panel the functions $\min \chi_{\Sigma}^{2}\left(\Omega_{\Lambda}\right)$ with distinct minima have some difference in their the $1 \sigma$ errors.

Estimations of $\Omega_{k}$ in tables IV-VI are calculated via the function $\min \chi_{\Sigma}^{2}\left(\Omega_{k}\right)=\min _{H_{0}, \Omega_{m}} \chi_{\Sigma}^{2}$. These graphs are shown in the top-right panel of figure 2 .

The panels in the second row in figure 2 demonstrate how parameters of a minimum point of the function $\chi_{\Sigma}^{2}$ with the fitting formula (10) depend on $H_{0}, \Omega_{m}, \Omega_{\Lambda}, \Omega_{k}$. In the left panel these coordinates are $\Omega_{m}$ and $\Omega_{\Lambda}$, but also the value $\Omega_{k}=1-\Omega_{m}-\Omega_{\Lambda}$ is drawn as the black dashed line. The optimal value $\Omega_{m}$ remains practically constant in contrast with $\Omega_{\Lambda}$ and $\Omega_{k}$. The graphs of $h\left(\Omega_{m}\right), \ldots, h\left(\Omega_{k}\right)$ in other panels show $h=H_{0} / 100$, where $H_{0}$ is the optimal value corresponding to the minimum point of $\chi_{\Sigma}^{2}$.

In other panels of figure 2 we present the results of calculations as level lines at $1 \sigma(68.27 \%), 2 \sigma$ $(95.45 \%)$ and $3 \sigma(99.73 \%)$ confidence levels for the functions $\chi^{2}\left(p_{1}, p_{2}\right)$ in planes of two parameters, if the third parameter is fixed. For example, the functions $\chi_{\Sigma}^{2}\left(\Omega_{m}, \Omega_{\Lambda}\right)$ for the fixed optimal value (26) of $H_{0}$ are shown in the bottom-left panel of figure 2 as red filled contours for the formula (10) and blue filled contours for Eq. (11). The corresponding level lines for $\chi_{S N}^{2}$ and for $\chi_{S N}^{2}+\chi_{H}^{2}$ are shown as black and green lines.

In other panels only $\chi_{\Sigma}^{2}$ filled contours for the cases (10) and (11) are compared for different pairs of parameters. Points of minima for the functions $\chi_{\Sigma}^{2}$ are marked as red (or blue) circles, we mentioned above, that they coincide for the variants (10) and (11). In two bottom-left panels the difference is in the fixed parameter $\left(\Omega_{\Lambda}\right.$ or $\left.\Omega_{k}\right)$ and in a choice of the foreground between the cases (10) and (11).

Our estimations of the $\Lambda \mathrm{CDM}$ parameters for two variants (10) and (11) of the fitting formula for $r_{d}$ are to be compared with the the following best fits for these model parameters from surveys of the Wilkinson Microwave Anisotropy Probe (WMAP) [8] and Planck Collaboration [9, 10] in table IV.

\begin{tabular}{||c||c|c||c|c|c||}
\hline \multicolumn{1}{||c||}{} & \multicolumn{2}{c||}{ This paper } & \multicolumn{3}{c||}{ WMAP, Planck surveys } \\
\hline & $r_{d}$ [10]) & $r_{d}$ [11]) & WMAP 9y [8] & Planck 13 [9] & Planck 15 [10] \\
\hline$H_{0}$ & $70.07 \pm 1.82$ & $70.07 \pm 1.27$ & $69.7 \pm 2.4$ & $67.3 \pm 1.2$ & $67.8 \pm 0.9$ \\
\hline$\Omega_{m}$ & $0.278 \pm 0.008$ & $0.278 \pm 0.009$ & $0.279 \pm 0.025$ & $0.314 \pm 0.02$ & $0.308 \pm 0.012$ \\
\hline$\Omega_{\Lambda}$ & $0.750_{-0.055}^{+0.051}$ & $0.750 \pm 0.034$ & $0.721 \pm 0.025$ & $0.686 \pm 0.025$ & $0.692 \pm 0.012$ \\
\hline$\Omega_{k}$ & $-0.028_{-0.048}^{+0.050}$ & $-0.028_{-0.034}^{+0.035}$ & $-0.0027_{-0.0038}^{+0.0039}$ & $-0.0005_{-0.0066}^{+0.0065}$ & $-0.005_{-0.017}^{+0.016}$ \\
\hline$\Omega_{b}$ & $0.044 \pm 0.004$ & - & $0.0463_{-0.0024}^{+0.024}$ & $0.0487_{-0.0017}^{+0.0018}$ & $0.0484_{-0.0013}^{+0.0014}$ \\
\hline
\end{tabular}

TABLE IV: Estimations of the $\Lambda$ CDM parameters.

One can also add the estimates for the fixed $r_{d}$ (9): $H_{0}=69.27 \pm 0.93 \mathrm{~km} \mathrm{~s}^{-1} \mathrm{Mpc}^{-1}, \Omega_{m}=$ $0.280 \pm 0.009, \Omega_{\Lambda}=0.732_{-0.055}^{+0.044}, \Omega_{k}=-0.012 \pm 0.045$ (corresponding to green dots in the top panels in figure 21). In the case (9) the $1 \sigma$ error for $H_{0}$ is smallest, because $d_{z}^{\text {th }}$ depends on $h$. 
We see that our estimations of the model parameters in table IV for the cases (10) and (11) are in good agreement with the WMAP estimates, but they are in $1 \sigma$ or $2 \sigma$ tension with the values of Planck Collaboration. On the other hand, all $H_{0}$ values in table IV have essential tension with the Hubble Space Telescope group [58] estimation: $H_{0}=73.8 \pm 2.4 \mathrm{~km} \mathrm{~s}^{-1} \mathrm{Mpc}^{-1}$.

The latter value was used as a prior in Refs. [55, 56] for describing Type Ia SNe, BAO and $H(z)$ data with the help of the $\Lambda \mathrm{CDM}, \mathrm{XCDM}$ and $\phi \mathrm{CDM}$ models. One may conclude, that for the $\Lambda \mathrm{CDM}$ this choice of $H_{0}$ was unsuccessful in comparison with another value $H_{0}=68 \mathrm{~km} \mathrm{~s}^{-1} \mathrm{Mpc}^{-1}$, chosen in Refs. [55, 56].

\section{MODIFIED CHAPLYGIN GAS}

In the model with modified Chaplygin gas (MCG) this gas has the following equation of state [41-47]

$$
p_{g}=w_{0} \rho_{g}-B \rho_{g}^{-\alpha}
$$

for its density $\rho_{g}$ as a part in the total density (16). MCG can unify dark matter and dark energy. If $w_{0}=0$ the MCG model with Eq. (27) is reduced to the model with generalized Chaplygin gas (GCG) with EoS [40, 59]

$$
p_{g}=-B \rho_{g}^{-\alpha}
$$

In our paper [39] the GCG model was applied to describing the observational data for Type Ia supernovae [3],$H(z)$ with 34 data points and BAO with 7 data points for $d_{z}(z)$. In this paper we consider the enlarged number of data points from tables II, III, the more general MCG model (27) (in comparison with the GCG case $w_{0}=0$ ) and also we calculate the function $\chi_{S N}^{2}(3)$ with the covariance matrix $C_{S N}$.

The MCG and GCG models are to be explored as two-component models with usual dust-like baryonic matter component $\rho_{b}$ and the Chaplygin gas component $\rho_{g}$ with EoS (27). In this case the total density (16) is

$$
\rho=\rho_{b}+\rho_{g}, \quad p_{b}=0 .
$$

However the first component $\rho_{b}$ and the corresponding fraction $\Omega_{b}(25)$ may include not only visible baryonic matter but also a part of cold dark matter with $\rho=\rho_{d m}$. Our practical applications of these models in Ref. [39] and in this paper (see the top-right panel of figure 3) demonstrate rather weak dependence of $\min \chi_{\Sigma}^{2}$ on $\Omega_{b}$ for the model assumption (11), but the strong $\Omega_{b}$ dependence for the fitting formula (10). This behavior resembles the $\Lambda$ CDM model, where separation of baryonic and cold dark matter in $\Omega_{m}$ appears only in Eq. (10). In the MCG and GCG models these matter fractions may also be mixed in their observational manifestations, so below we did not use $\Omega_{b}$ as an usual free parameter of the theory, but fix its fiducial value (12) in the main part this research (except for calculations, presented in right panels if figures 3 and 4).

Equation (19) for the MCG model (27) is integrable, so the analog of Eq. (20) for this model is [41-47]

$$
\frac{H^{2}}{H_{0}^{2}}=\Omega_{b}\left(\frac{a}{a_{0}}\right)^{-3}+\Omega_{k}\left(\frac{a}{a_{0}}\right)^{-2}+\left(1-\Omega_{b}-\Omega_{k}\right)\left[B_{s}+\left(1-B_{s}\right)\left(\frac{a}{a_{0}}\right)^{-3\left(1+w_{0}\right)(1+\alpha)}\right]^{1 /(1+\alpha)} .
$$

Here the dimensionless parameter $B_{s}=B \rho_{0}^{-1-\alpha} /\left(1+w_{0}\right)$ is used instead of $B$. Thus in the MCG model (27) we have 6 independent parameters: $H_{0}, \Omega_{b}, \Omega_{k}, w_{0}, \alpha$ and $B_{s}$. Naturally, the GCG model has 5 parameters: the same set without $w_{0}$. 
In the MCG model the parameter $\Omega_{m}$ from its formal definition (21) equals $\Omega_{m}=1-\Omega_{k}$ in accordance with $\Omega_{\Lambda}=0$ and Eq. (22). However, the expression $A(z)$ (7) has the factor $\sqrt{\Omega_{m}}$, so one should use the effective value $\Omega_{m}^{e f f}$ in any model. If we compare the early universe limit $z \gg 1$ in the MCG equation (30) with the $\Lambda \mathrm{CDM}$ equation (20), we obtain the effective value [45 47]:

$$
\Omega_{m}^{e f f}=\Omega_{b}+\left(1-\Omega_{b}-\Omega_{k}\right)\left(1-B_{s}\right)^{1 /(1+\alpha)} .
$$

But for the majority of observational data in Ref. [3] and tables [I, [II redshifts are $0<z<1$, so to describe correctly these data we have to consider the present time limit of Eq. (30). If we compare limits of the right hand sides of Eqs. (20) and (30) at $z \rightarrow 0$ or $\mathcal{A} \rightarrow 0$, we obtain another effective value 39]

$$
\Omega_{m}^{e f f}=\Omega_{b}+\left(1-\Omega_{b}-\Omega_{k}\right)\left(1-B_{s}\right)\left(1+w_{0}\right) .
$$

Expressions (311) and (32) and their contributions in $\chi_{B A O}^{2}$ and $\chi_{\Sigma}^{2}$ were compared in Ref. [39]. In this paper we use below Eq. (32) and its analogs for other models.

Figure 3 shows how the MCG model (27) describes the observational data from Ref. [3] and tables II, III in comparison with the GCG model (28). Notations are the same as in figure 2. In the top line panels red solid lines and blue dashed lines denote graphs of $\min \chi_{\Sigma}^{2}$ for the MCG model with expressions (10) and (11) correspondingly. The similar functions for the GCG model are shown as orange dash-dotted lines, if $r_{s}\left(z_{d}\right)$ is calculated with the formula (10) and as violet dashed lines for the case (11).

These graphs in the top line describe how $\chi_{\Sigma}^{2}$ for the mentioned models depends on one chosen model parameter: $H_{0}, \Omega_{k}, w_{0}, \alpha, \Omega_{b}$. All these curves determine the optimal values and errors of the model parameters in tables $\mathrm{V}$, VI. The second row panels of figure 3 correspond to panels in the top line and present dependencies of coordinates of minima points on $H_{0}, \ldots, \Omega_{b}$ for $\chi_{\Sigma}^{2}$ in the MCG model with the fitting formula (10).

In the top-right panel of figure 3 the value $\min \chi_{\Sigma}^{2}$ for the MCG model means the minimum

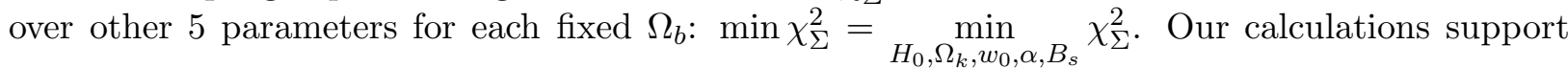
the previous conclusion [39] about weak dependence of $\min \chi_{\Sigma}^{2}$ on $\Omega_{b}$ for the case (11) with $r_{d}=$ $\left(r_{d} h\right)_{f i d} \cdot h^{-1}$ as for the the fixed value $r_{d}$ (9) for both models. This is connected with possible mixing of baryonic and cold dark matter in $\Omega_{b}$. However, for and the fitting formula (10) we see the sharp dependence of $\min \chi_{\Sigma}^{2}$ on $\Omega_{b}$; for both MCG and GCG models this picture coincides with behavior of the $\Lambda$ CDM model in figure 1. As mentioned above, we will not use $\Omega_{b}$ as an usual free model parameter and we fix its $\Lambda$ CDM fiducial value (12). It is interesting that for the MCG and GCG models this value $\Omega_{b} \simeq 0.044 \pm 0.004$ is very close and practically coincides with the $\Lambda$ CDM fiducial value of this parameter.

In figure 3 the parameter $\Omega_{b}$ is varied only in 3 right panels, in other 12 panels this value is fixed in the form (12). In particular, the value $\min \chi_{\Sigma}^{2}\left(H_{0}\right)$ for the MCG model in the top-left panel is the minimum over 4 parameters $\min _{\Omega_{k}, w_{0}, \alpha, B_{s}} \chi_{\Sigma}^{2}$ with fixed $\Omega_{b}=0.044$. The similar picture takes place, when we study dependence of these minimal functions on $\Omega_{k}, w_{0}, \alpha$, for example, $\min \chi_{\Sigma}^{2}\left(\Omega_{k}\right)=\min _{H_{0}, w_{0}, \alpha, B_{s}} \chi_{\Sigma}^{2}$. For the GCG model with $w_{0}=0$ these minima (orange dash-dotted lines and violet dashed lines) are taken over 3 remaining parameters.

The graphs of $\min \chi_{\Sigma}^{2}\left(H_{0}\right)$ in the top-left panel with two expressions for $r_{s}\left(z_{d}\right)$ resemble the $\Lambda \mathrm{CDM}$ case in figure 2 for both MCG and GCG models. For all three considered models this curve for the case (11) is slightly more sharp than for the formula (10), this behavior may be seen in tables V, VI, where all models are compared. Unlike the $\Lambda$ CDM model, for the MCG and GCG models we have different optimal values of $H_{0}$ (and also of $\Omega_{k}, \alpha$ and other parameters) in the cases (10) and (111). 

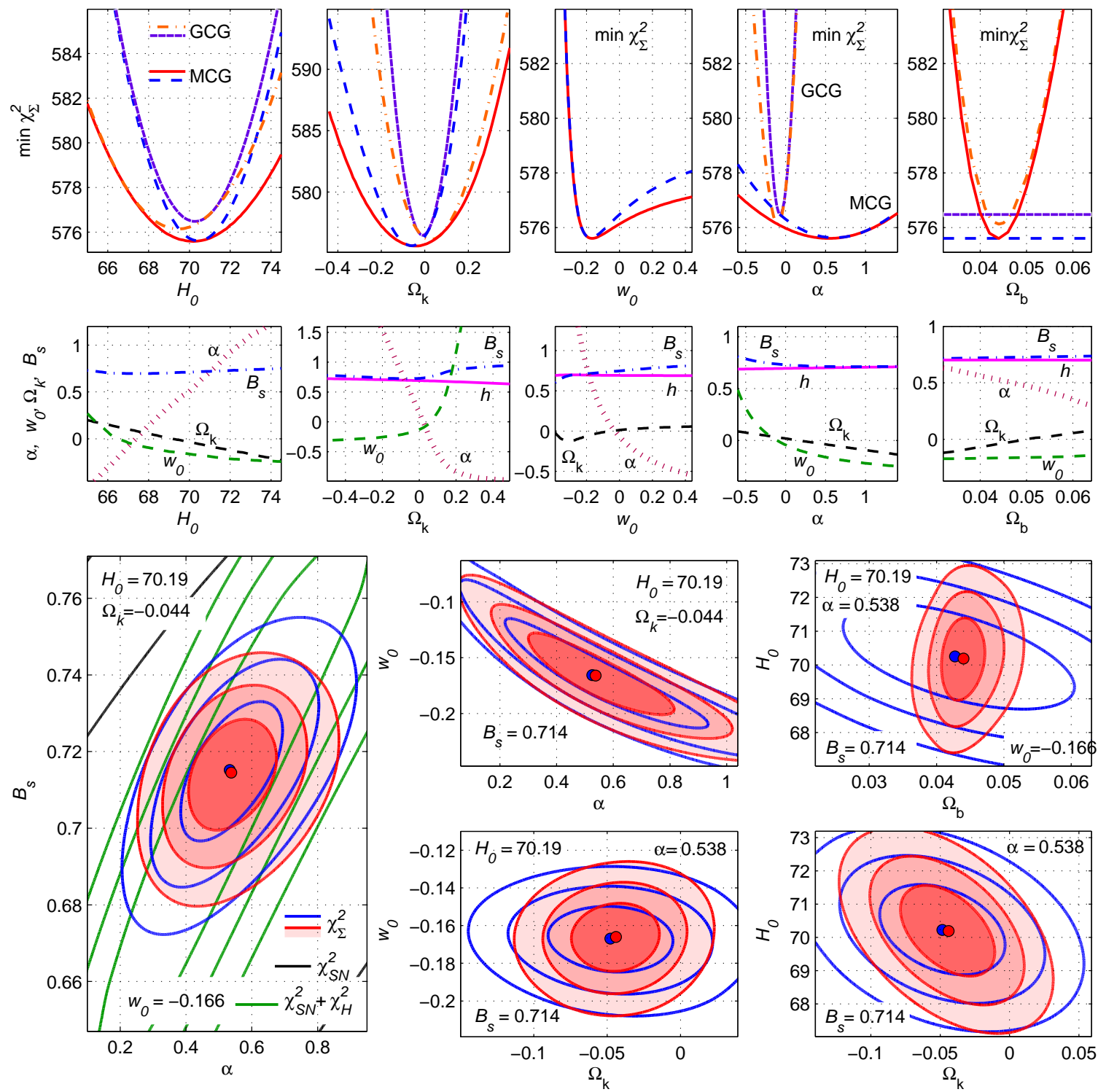

FIG. 3: For the MCG model dependencies of $\min \chi_{\Sigma}^{2}$ on one chosen parameter $H_{0}, \Omega_{k}, w_{0}, \alpha, \Omega_{b}$ are presented for the fitting formulae (10) (red solid lines) and (11) (blue dashed lines) in comparison with the GCG model (orange dash-dotted lines and violet dashed lines for these two choices of $r_{d}$ ). In the second row there are correspondent MCG parameters of a minimum point for $\chi_{\Sigma}^{2}$ with Eq. (10). In other panels for the MCG model $1 \sigma, 2 \sigma, 3 \sigma$ level lines are drawn in the planes of 2 parameters (other parameters are fixed). Notations correspond to figure 2 .

Obviously that for the GCG model the graphs of $\chi_{\Sigma}^{2}$ in the top panels of figure 3 lie higher, then the correspondent MCG graphs. These curves converge at points, where the optimal value of $w_{0}$ equals zero in the panel below.

The graphs of $\min \chi_{\Sigma}^{2}\left(\Omega_{k}\right)$ in the second top panel are asymmetric, for $\Omega_{k}>0$ these values for the GCG and MCG models are close, but for $\Omega_{k}<0$ the curves for these models diverge for both variants of $r_{s}\left(z_{d}\right)$. In the third top panel we have asymmetric dependence of these minima on $w_{0}$ for the MCG model.

Dependence od $\min \chi_{\Sigma}^{2}$ on $\alpha$ is essentially different for the considered models: for the GCG model these curves have sharp minima, in particular, for the case (10) at $\alpha \simeq-0.1$ with $\sigma \simeq 0.1$, 
but for the MCG model the dependence is rather smooth, the minimum at $\alpha \simeq 0.54$ has $\sigma \simeq 0.9$. These results are also presented in table $\mathrm{V}$, correspondent values for the function (11) are in table VI. One may conclude that changes of the parameter $w_{0}$ may compensate changes of $\alpha$.

In 5 bottom panels of figure 3 for the MCG model we present $1 \sigma, 2 \sigma$ and $3 \sigma$ level lines in the planes of 2 model parameters (in notations of figure 2), in particular, red filled contours denote levels of $\chi_{\Sigma}^{2}$ with the fitting formula (10). For this case in the bottom-left panel in the $\left(\alpha, B_{s}\right)$ plane contours for $\chi_{S N}^{2}$ (black) and $\chi_{S N}^{2}+\chi_{H}^{2}$ (green lines) are also shown. Contours for $\chi_{\Sigma}^{2}$ with the formula (11) in all panels are drawn as blue lines. Other model parameters are fixed and shown in the panels and in table $\mathrm{V}$, they are optimal for the fitting formula (10) (but not optimal for the case (11)).

\begin{tabular}{||l||c||c|c|l||}
\hline Model & $\min \chi_{\Sigma}^{2}$ & $H_{0}$ & $\Omega_{k}$ & other parameters \\
\hline$\Lambda$ CDM & 577.39 & $70.07 \pm 1.82$ & $-0.028_{-0.048}^{+0.050}$ & $\Omega_{m}=0.278 \pm 0.008, \Omega_{\Lambda}=0.750_{-0.055}^{+0.051}$ \\
\hline GCG & 576.13 & $69.46 \pm 1.88$ & $0.026_{-0.069}^{+0.075}$ & $\alpha=-0.100_{-0.098}^{+0.090}, B_{s}=0.738_{-0.022}^{+0.024}$ \\
\hline MCG & 575.60 & $70.19 \pm 2.15$ & $-0.044 \pm 0.122$ & $\begin{array}{l}\alpha=0.538_{-0.893}^{+0.902}, B_{s}=0.714_{-0.031}^{+0.033} \\
w_{0}=-0.166_{-0.089}^{+0.322}\end{array}$ \\
\hline $\begin{array}{l}\text { EoS } \\
(34)\end{array}$ & 575.15 & $70.28_{-2.10}^{+2.22}$ & $-0.045_{-0.094}^{+0.102}$ & $\begin{array}{l}p_{0}=-0.904_{-0.248}^{+0.256}, \beta=-0.042_{-0.038}^{+0.041} \\
w_{0}=0.183 \pm 0.222\end{array}$ \\
\hline
\end{tabular}

TABLE V: Models and $1 \sigma$ estimates of model parameters, if $r_{s}\left(z_{d}\right)$ has the form (10); $\Omega_{b}=0.044$.

\begin{tabular}{||l||c||c|c|l||}
\hline Model & $\min \chi_{\Sigma}^{2}$ & $H_{0}$ & $\Omega_{k}$ & other parameters \\
\hline$\Lambda$ CDM & 577.39 & $70.07 \pm 1.27$ & $-0.028_{-0.034}^{+0.035}$ & $\Omega_{m}=0.278 \pm 0.009, \Omega_{\Lambda}=0.750_{-0.034}^{+0.033}$ \\
\hline GCG & 576.48 & $70.27 \pm 1.28$ & $-0.009 \pm 0.040$ & $\alpha=-0.069 \pm 0.074, B_{s}=0.753 \pm 0.013$ \\
\hline MCG & 575.61 & $70.44 \pm 1.30$ & $-0.060_{-0.075}^{+0.068}$ & $\begin{array}{l}\alpha=0.613_{-0.744}^{+0.80}, B_{s}=0.716_{-0.033}^{+0.058}, \\
w_{0}=-0.176_{-0.078}^{+0.447}\end{array}$ \\
\hline $\begin{array}{l}\text { EoS } \\
(\underline{34})\end{array}$ & 575.14 & $70.42 \pm 1.30$ & $-0.048_{-0.048}^{+0.054}$ & $\begin{array}{l}p_{0}=-0.928_{-0.230}^{+0.242}, \beta=-0.045_{-0.039}^{+0.043}, \\
w_{0}=0.205_{-0.180}^{+0.187}\end{array}$ \\
\hline
\end{tabular}

TABLE VI: Models and $1 \sigma$ estimates of model parameters with $r_{s}\left(z_{d}\right)$ from Eq. (11); $\Omega_{b}=0.044$.

For the GCG model (28) the values in table VI are close to our previous estimations [39] $H_{0}=70.093 \pm 0.369, \Omega_{k}=-0.019 \pm 0.045, \alpha=-0.066_{-0.074}^{+0.072}, B_{s}=0.759_{-0.016}^{+0.015}$ with 7 and 34 data points for $d_{z}(z)$ and $H(z)$ correspondingly.

These estimates for the MCG model should be compared with similar results for this model in papers [45-47]. The authors of Ref. [45] for the flat model with $\Omega_{k}=0$ described the observational data with 557, 15 and 2 data points correspondingly for supernovae, $H(z)$ and BAO, but they also included the cluster X-ray gas mass fraction data. Their $1 \sigma$ estimations $H_{0}=70.711_{-3.142}^{+4.188}$ and $B_{s}=0.7788_{-0.0723}^{+0.0736}$ are more wide then ours in tables $\mathrm{V}$, VI, however $\alpha=0.1079_{-0.2539}^{+0.3397}$ and the narrow box $w_{0}=0.00189_{-0.00756}^{+0.00583}$ lie inside our $1 \sigma$ estimates.

In Refs. [46, 47] the MCG model with $\Omega_{k}=0$ is applied for describing $12 H(z)$ data points, 11 points for the growth function $f=d \log \delta / d \log a$ of the large scale structures, 17 points for $\sigma_{8}(z)$ and also in Ref. [47] observations of supernovae and 1 data point for BAO. The authors did not demonstrate their estimates for $H_{0}$, but noted ambiguously " $\chi^{2}$ function for the background test is minimized by the present Hubble value predicted by WMAP7". The best fit values of other parameters in Ref. [47] are $w_{0}=0.005, \alpha=0.19, B_{s}=0.825$ with errors, calculated for pairs of these parameters. Only the estimate for $B_{s}$ is in tension with our results in tables V. VI. 


\section{MODEL WITH QUADRATIC EQUATION OF STATE} $48-53]$

It is interesting to compare the MCG model and the model with quadratic equation of state

$$
p_{g}=\tilde{p}_{0}+w_{0} \rho_{g}+\tilde{\beta} \rho_{g}^{2},
$$

because both models have 6 parameters: $H_{0}, \Omega_{k}, \Omega_{b}$ and 3 parameters in the EoS (27) or (33). It is convenient to use the critical density $\rho_{c r}=3 H_{0}^{2} /(8 \pi G)$, introduce the dimensionless parameters $p_{0}=\tilde{p}_{0} / \rho_{c r}, \beta=\tilde{\beta} \rho_{c r}$ instead of $\tilde{p}_{0}, \tilde{\beta}$ and rewrite Eq. (133) in the form

$$
p_{g}=p_{0} \rho_{c r}+w_{0} \rho_{g}+\beta \rho_{g}^{2} / \rho_{c r} .
$$

Similarly to the GCG and MCG models the model with quadratic EoS (33) or (34) has the analytical general solution of Eq. (19) [50]

$$
\frac{\rho_{g}}{\rho_{c r}}= \begin{cases}\frac{1}{2 \beta}\left[\frac{\Gamma-\sqrt{|\Delta|} \tan \left(\frac{3}{2} \sqrt{|\Delta|} \mathcal{A}\right)}{1+|\Delta|^{-1 / 2} \tan \left(\frac{3}{2} \sqrt{|\Delta|} \mathcal{A}\right)}-1-w_{0}\right], & \Delta<0, \\ \frac{1}{2 \beta}\left[\left(\frac{3}{2} \mathcal{A}+1 / \Gamma\right)^{-1}-1-w_{0}\right], & \Delta=0, \\ \frac{\rho_{-}\left(\Omega_{m}-\rho_{+}\right)\left(a / a_{0}\right)^{-3 \sqrt{\Delta}}-\rho_{+}\left(\Omega_{m}-\rho_{-}\right)}{\left(\Omega_{m}-\rho_{+}\right)\left(a / a_{0}\right)^{-3 \sqrt{\Delta}}-\Omega_{m}+\rho_{-}}, & \Delta>0,\end{cases}
$$

depending on a sign of the discriminant $\Delta=\left(1+w_{0}\right)^{2}-4 \beta p_{0}$. Here

$$
\Omega_{m}=1-\Omega_{k}-\Omega_{b}, \quad \Gamma=2 \beta \Omega_{m}+1+w_{0}, \quad \rho_{ \pm}=\frac{-1-w_{0} \pm \sqrt{\Delta}}{2 \beta}, \quad \mathcal{A}=\log \frac{a}{a_{0}} .
$$

The equation (18) for this model is reduced to the form

$$
\frac{H^{2}}{H_{0}^{2}}=\left(\frac{d \mathcal{A}}{d \tau}\right)^{2}=\frac{\rho_{g}}{\rho_{c r}}+\Omega_{b} e^{-3 \mathcal{A}}+\Omega_{k} e^{-2 \mathcal{A}} .
$$

We solve this equation numerically from the present time initial condition $\left.\mathcal{A}\right|_{\tau=1}=0$ "to the past". We can use analytical solution (35) or solve Eq. (19) numerically, these approaches are equivalent.

For the model (34) the effective value

$$
\Omega_{m}^{e f f}=\Omega_{b}+p_{0}+\Omega_{m}\left(1+w_{0}+\beta \Omega_{m}\right)
$$

is calculated in the $z \rightarrow 0$ or $\mathcal{A} \rightarrow 0$ limit similarly to the MCG model.

The model with quadratic $\operatorname{EoS}($ (34) $)$ in the domain $\beta>0$ may have the following singularity in the past: when $t \rightarrow t_{*}+0$, density grows to infinity, but the scale factor remains finite and nonzero: $\lim _{t \rightarrow t_{*}} \rho_{g}=\infty, \lim _{t \rightarrow t_{*}} a=a\left(t_{*}\right) \neq 0$. This behavior resembles the Type III finite-time future singularity from the classification [36, 37]. In Ref. [53] the author did not see these singularities, because he considered only negative values $\beta=-\left(w_{0}+1\right) / \rho_{P}$.

In the bottom-right panel of figure 4 we present the example of singular solution as the red dashed line for $a(\tau) / a_{0}$ and the black dash-dotted line for $0.01 \cdot \rho(\tau) / \rho_{c r}$. This singularity is compared with the regular solution (the blue solid line for $a(\tau) / a_{0}$ ) with the optimal values of model parameters from table $\mathrm{V}$. For the singular solution in this panel $\beta=0.02$, but other model parameters are from table $\mathrm{V}$.

We have to exclude these nonphysical singular solutions, for this purpose we can use different approaches. The simplest way is to add the penalty contribution in $\chi_{\Sigma}^{2}$ in the form

$$
\Delta \chi_{\Sigma}^{2}=P_{1}\left[\exp \left(P_{2} a\left(t_{*}\right) / a_{0}\right)-1\right] .
$$


The function (38) with $P_{1}=0.3$ and $P_{2}=20$ successfully helps to avoid this singularity. For regular solutions $a\left(t_{*}\right)=0$ and the contribution (38) vanishes. In the top panels of figure 4 functions min $\chi_{\Sigma}^{2}$ with the contribution (38) are shown as solid red lines for the formula (10) and as blue dashed lines for $r_{d}=\left(r_{d} h\right)_{f i d} \cdot h^{-1}$; for the case without penalty (38) these graphs are dotted lines of the correspondent color: red for the formula (10) and blue for (11).

More natural method to eliminate nonphysical singular solutions is to include early time parameters (for example, $z_{d}$ ) into consideration. Unfortunately, both equations (10) and (11) or $r_{s}\left(z_{d}\right)$ are insensitive to the mentioned singularities. So we can take into account the cosmic microwave background (CMB) constraints, in particular, for the values [7]

$$
\mathbf{v}=\left(\omega_{b}, \omega_{c b}, D_{M}(1090) / r_{d}\right),
$$

where $\omega_{i}=\omega_{i} h^{2}, D_{M}(z)=D_{L}(z) /(1+z)$. If we calculate the vector (39), compare it with the estimation [7, 8] $\Delta \mathbf{v}=\mathbf{v}-(0.02259,0.1354,94.51)$ and add the corresponding term to the $\chi_{\Sigma}^{2}$ function (24), we obtain

$$
\chi_{\Sigma+}^{2}=\chi_{\Sigma}^{2}+\Delta \mathbf{v} \cdot C_{C M B}^{-1}(\Delta \mathbf{v})^{T} .
$$

Here the covariance matrix from Ref. [7] contains $c_{11}=2.864 \cdot 10^{-7}, c_{12}=-4.809 \cdot 10^{-7}$, $c_{13}=-1.111 \cdot 10^{-5}, c_{22}=1.908 \cdot 10^{-5}, c_{23}=-7.495 \cdot 10^{-6}, c_{33}=0.02542$.

Graphs of one parameter functions $\min \chi_{\Sigma+}^{2}$ with the CMB contribution for the case (10) are also presented in the top panels of figure 4 as black dash-dotted lines.

We demonstrate in figure 4 how the model (34) is effective in describing the observational data from Ref. [3] and tables [I, III. Notations are the same as in figure 3, Here we also fix the value (12) $\Omega_{b}=0.044$ (except for 2 top-right panels) and do not use $\Omega_{b}$ as a fitting parameter. The dependence $\min \chi_{\Sigma}^{2}\left(\Omega_{b}\right)=\min _{H_{0}, \Omega_{k}, w_{0}, p_{0}, \beta} \chi_{\Sigma}^{2}$ (shown in the top-right panel) is rather weak for the case (11) $r_{d}=\left(r_{d} h\right)_{f i d} \cdot h^{-1}$, but it is essential for the formula (10) and for the function min $\chi_{\Sigma+}^{2}$. In the case $\min \chi_{\Sigma}^{2}$ with Eq. (10) (the red curve) the dependence $\min \chi_{\Sigma}^{2}\left(\Omega_{b}\right)$ is very close to the correspondents functions for the $\Lambda \mathrm{CDM}, \mathrm{GCG}, \mathrm{MCG}$ models in figures 1, 3. its minimum is also at $\Omega_{b} \simeq 0.044 \pm 0.004$.

In the top line panels of figure 4 we draw graphs of $\chi_{\Sigma}^{2}$ minima depending on one model parameter $\left(H_{0}, \Omega_{k} \ldots\right)$ for the model (34) with the penalty function (38) as solid red lines for the formula (10) and as blue dashed lines for the case (11). Correspondent lines without contribution (38) are shown as dots of the same color. In other words, if we calculate min $\chi_{\Sigma}^{2}$ only for (physical) regular solutions, we obtain the solid or dashed lines; for dots we also include singular solutions without physical interpretation. These lines coincide in domains where best values of $\beta$ are negative and corresponding solutions are regular. One can see in figure 4 that optimal values of model parameters correspond to regular solutions in both considered cases (10) (table V) and (11) (table VI).

Minima of $\chi_{\Sigma}^{2}$ and $\chi_{\Sigma+}^{2}$ here have the same sense as in figure 3 for the MCG model, in particular, in the top-left panel $\min \chi_{\Sigma}^{2}\left(H_{0}\right)=\min _{\Omega_{k}, w_{0}, p_{0}, \beta} \chi_{\Sigma}^{2}$. Dependence of this minimum on $H_{0}$ for two formulas for $r_{s}\left(z_{d}\right)$ resembles other considered models (compare with figures 2, 3): the graph for the case (11) is more sharp, than for Eq. (10), it corresponds to larger optimal value of $H_{0}$ and smaller $1 \sigma$ error in table VI than the values in table V. For other panels of the top line in figure 4 dependence of $\min \chi_{\Sigma}^{2}$ on parameters $\Omega_{k}, w_{0}$ and $p_{0}$ results in the correspondent the $1 \sigma$ estimates in tables $\mathrm{V}, \mathrm{VI}$,

The functions min $\chi_{\Sigma+}^{2}$ with the CMB contribution (40) depending on one parameter are shown as black dash-dotted lines in the top line panels. We see that the absolute minimum of this function is $\min \chi_{\Sigma+}^{2} \simeq 576.16$, in a bit exceeds the corresponding value $\min \chi_{\Sigma}^{2} \simeq 575.15$ for the case (10) 

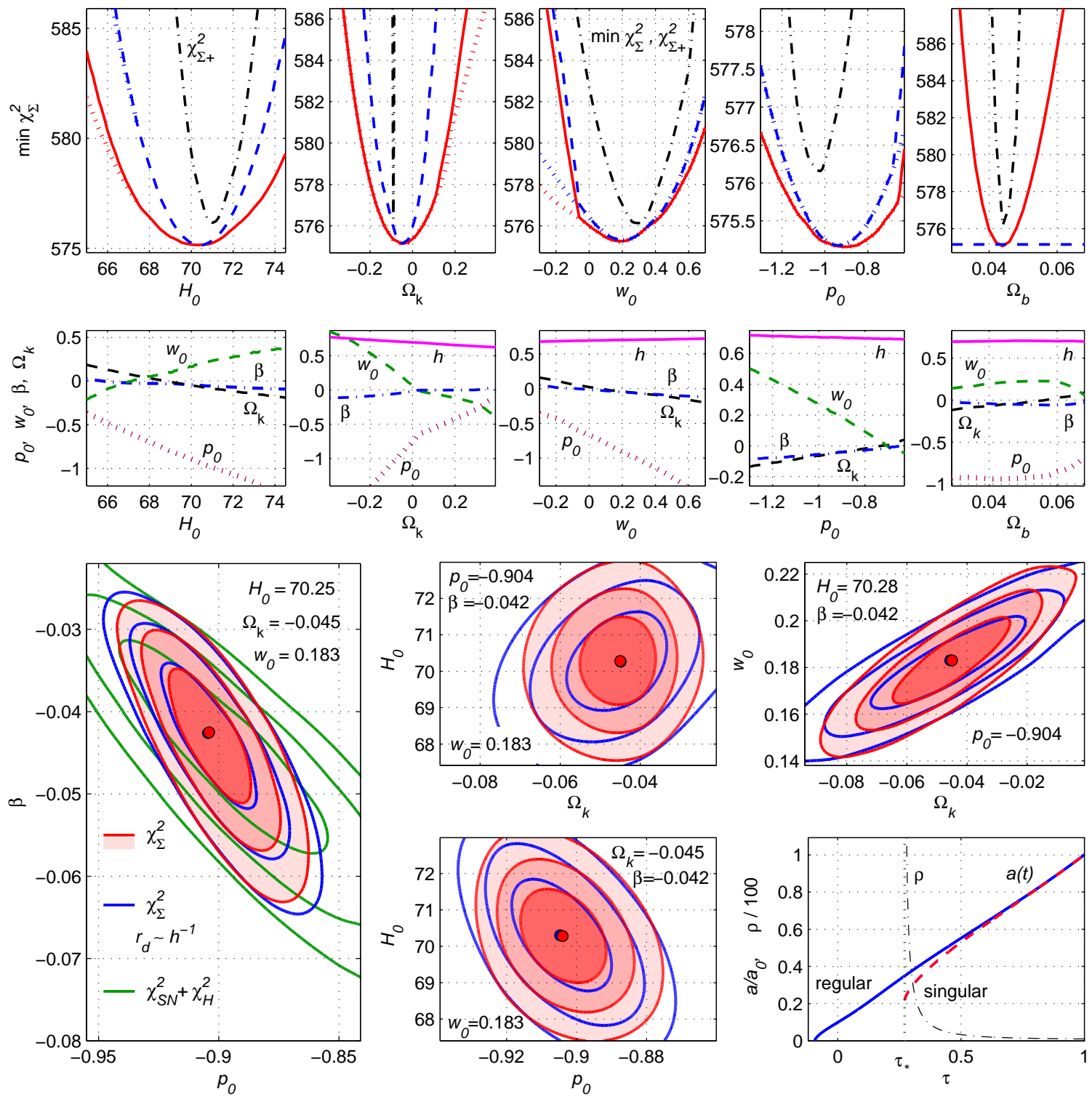

FIG. 4: For the model with quadratic EoS (34) one parameter dependencies of min $\chi_{\Sigma}^{2}$ with $r_{s}\left(z_{d}\right)$ in the forms (10) (red solid lines) and (11) (blue dashed lines), of min $\chi_{\Sigma}^{2}$ with CMB contribution and Eq. (10) (black dash-dotted lines) and also coordinates of minima points, level lines are presented in notations of figure 3. In the bottom-right panel scale factors $a / a(0)$ for the regular solution (the blue solid line) and the singular solution (the red dashed line) are shown.

in table V] However, the CMB contribution in the form (40) works as a very narrow filter for some model parameters, in particular, for the considered model with EoS (34) this contribution rigidly constrains the value $\Omega_{k}$, so we have the $\chi_{\Sigma+}^{2}$ estimation $\Omega_{k}=-0.090 \pm 0.001$. The curve, corresponding to this narrow range, is shown in the second top panel of figure 4, it is too narrow, so it looks like a vertical black segment.

The $\chi_{\Sigma+}^{2}$ estimations of other parameters are also more narrow (see the top line in figure 4), than for both cases of $\chi_{\Sigma}^{2}$, it is connected with restrictions for $\Omega_{k}$ and other correspondent parameters for other models. Some of these restrictions look like artificial od connected with the concrete choice of the CMB vector (39). So further we consider the functions $\min \chi_{\Sigma}^{2}$ without the CMB 
contribution (40) with the fitting formula (10) as the most reliable indicator.

Panels in the second row of figure 4 correspond the upper panels for the function min $\chi_{\Sigma}^{2}$ with the fitting formula (10) with the penalty contribution (38). They demonstrate evolution of coordinates of the minimal point if we vary the chosen parameter. In 4 bottom panels functions $\chi^{2}$ depend on two model parameters $\left(p_{0}, \beta ; p_{0}, H_{0} ; \Omega_{k}, H_{0} ; \Omega_{k}, w_{0}\right)$ when other parameters are fixed with their optimal values from table $\mathrm{V}$, Red filled contours denote the case (10), blue lines corresponds to (11). These level lines demonstrate similarity with the MCG model in figure 3, but for the model (34) we have alternative behavior in the $\left(\Omega_{k}, H_{0}\right)$ plane. One should emphasize that all $1 \sigma, 2 \sigma$ and $3 \sigma$ level lines for $\chi_{\Sigma}^{2}$ in figure 4 and optimal values of the model parameters in table V] VI lie in the domain with regular solutions of the quadratic EoS model (34).

\section{CONCLUSION}

In this paper the Type Ia supernovae observational data from Ref. [3] and estimations of BAO parameters and $H(z)$ from tables II, III are described with the models $\Lambda$ CDM, GCG (28), MCG (27) and the model with quadratic EoS (34). Two approaches in estimation of the sound horizon scale $r_{s}\left(z_{d}\right)$ are used and compared: the fitting formula (10) with results, tabulated in table $\mathrm{V}$ and the expression (11) $r_{d}=\left(r_{d} h\right)_{f i d} \cdot h^{-1}$ (table VI. Optimal values of model parameters with $1 \sigma$ errors in these tables are calculated via one-parameter distributions (figures 2-4).

We also considered the CMB contribution in the form [7] (40), the results for the model with EoS (34) are shown in figure 4. However, this contribution appeared to be too sensitive and restrictive for some model parameters, in particular, $\Omega_{k}$ (or $\Omega_{\Lambda}$ for the $\Lambda$ CDM model). So we consider the $\chi_{\Sigma}^{2}$ estimations of model parameters in tables $\left.\mathrm{V}\right]$ and $\mathrm{VI}$ as more reliable.

It is interesting that predictions of the $\Lambda$ CDM, GCG, MCG models and the model with quadratic EoS for $\Omega_{b}$ are very close $\left(\Omega_{b}=0.044 \pm 0.004\right)$, if we adopt the fitting formula (10) for $r_{s}\left(z_{d}\right)$. We use this fact and do not consider $\Omega_{b}$ as an usual model parameter and fix its value in the form (12). One should note, that predictions of different models for $H_{0}$ and $\Omega_{k}$ in tables $\mathrm{V}$ and VI are also rather close.

Absolute minima of $\chi_{\Sigma}^{2}$ with the formula (10) in table $V$ differ from the correspondent minima of in table VI with Eq. (11), but the hierarchy of all considered models is the same in these tables. In particular, the absolute minimum of $\chi_{\Sigma}^{2}$ in table $\mathrm{V}$ vary from the worst value 577.39 for the $\Lambda \mathrm{CDM}$ to the best result 575.15 for the model with quadratic EoS (34). Note that the advantage of the MCG model in comparison with GCG is larger in the case (11) in table VI.

However, effectiveness of a model essentially depends on its number $N_{p}$ of model parameters (degrees of freedom). This number is used in model selection statistics, in particular, in the following Akaike information criterion [57, 60]

$$
A I C=\min \chi_{\Sigma}^{2}+2 N_{p} .
$$

If we fix the value $\Omega_{b}$ in the form (12) for the models GCG, MCG and with EoS (34) and do not use this parameter as a degree of freedom, we will have the numbers $N_{p}$ and $A I C$ for $\chi_{\Sigma}^{2}$ from table $\mathrm{V}$ for the considered models tabulated here in table VII

This information criterion works against models with large $N_{p}$ and adds arguments in favor of the $\Lambda$ CDM model.

\section{Acknowledgments}

The work is supported by the Ministry of education and science of Russia, grant No. 1686 . The author is grateful to S. D. Odintsov and E. G. Vorontsova for useful discussions, and to two 


\begin{tabular}{||l||c|c|c||}
\hline Model & $\min \chi_{\Sigma}^{2}$ & $N_{p}$ & $A I C$ \\
\hline$\Lambda$ CDM & 577.39 & 3 & 583.39 \\
\hline GCG & 576.13 & 4 & 584.13 \\
\hline MCG & 575.60 & 5 & 585.60 \\
\hline EoS (34) & 575.15 & 5 & 585.15 \\
\hline
\end{tabular}

TABLE VII: Akaike information criterion for the models.

unknown referees for valuable advices.

[1] A. G. Riess et al., Astron. J. 116 (1998) 1009, arXiv:astro-ph/9805201.

[2] S. Perlmutter et al., Astrophys. J. 517 (1999) 565, arXiv:astro-ph/9812133.

[3] N. Suzuki et al., Astrophys. J. $\mathbf{7 4 6}$ (2012) 85, arXiv:1105.3470 [astro-ph.CO]; http://supernova.lbl.gov/Union/.

[4] D. H. Weinberg et al., Phys. Rep. 530, (2013) 87, arXiv: 1201.2434 [astro-ph.CO].

[5] D. J. Eisenstein et al., Astrophys. J. 633 (2005) 560, arXiv:astro-ph/0501171.

[6] D. J. Eisenstein and W. Hu, Astrophys. J. 496 (1998) 605, arXiv:astro-ph/9709112.

[7] E. Aubourg et al., Phys. Rev. D 92 (2015) 123516, arXiv:1411.1074 [astro-ph.CO].

[8] WMAP collaboration, G. Hinshaw et al., Astrophys. J. Suppl. 208 (2013) 19, arXiv:1212.5226 [astroph.CO].

[9] Planck Collaboration, P. A. R. Ade et al. Astron. Astrophys. 571 (2014) A16, arXiv:1303.5076 [astroph.CO].

[10] Planck Collaboration, P. A. R. Ade et al. arXiv:1502.01589 [astro-ph.CO].

[11] J. Simon, L. Verde and R. Jimenez, Phys. Rev. D 71 (2005) 123001, arXiv:astro-ph/0412269.

[12] D. Stern, R. Jimenez, L. Verde, M. Kamionkowski and S. A. Stanford, J. Cosmol. Astropart. Phys. 02 (2010) 008, arXiv:0907.3149 [astro-ph.CO].

[13] M. Moresco et al., J. Cosmol. Astropart. Phys. 8 (2012) 006, arXiv:1201.3609 [astro-ph.CO].

[14] C. Zhang et al., Res. Astron. Astrophys. 14 (2014) 1221, arXiv:1207.4541 [astro-ph.CO].

[15] M. Moresco, arXiv:1503.01116 [astro-ph.CO].

[16] E. Gaztañaga, A. Cabre, L. Hui, Mon. Not. Roy. Astron. Soc. 399(3) (2009) 1663. arXiv:0807.3551 [astro-ph].

[17] C. Blake et al., Mon. Not. Roy. Astron. Soc. 425(1) (2012) 405, arXiv:1204.3674 [astro-ph.CO].

[18] N. G. Busca et al., Astron. and Astrop. 552 (2013) A96 arXiv:1211.2616 [astro-ph.CO].

[19] C-H. Chuang and Y. Wang, Mon. Not. Roy. Astron. Soc. 435(1) (2013) 255, arXiv:1209.0210 [astroph.CO].

[20] C-H. Chuang et al., Mon. Not. Roy. Astron. Soc. 433(4) (2013) 3559, arXiv:1303.4486 [astro-ph.CO].

[21] L. Anderson et al., Mon. Not. Roy. Astron. Soc. 439(1) (2014) 83, arXiv:1303.4666 [astro-ph.CO].

[22] L. Anderson et al., Mon. Not. Roy. Astron. Soc. 441 (2014) 24, arXiv:1312.4877 [astro-ph.CO].

[23] A. Oka et al., Mon. Not. Roy. Astron. Soc. 439(3) (2014) 2515, arXiv:1310.2820 [astro-ph.CO].

[24] A. Font-Ribera et al., J. Cosmol. Astropart. Phys. 05 (2014) 027, arXiv:1311.1767 [astro-ph.CO].

[25] T. Delubac et al., Astron. and Astrop. 574 (2015) A59, arXiv:1404.1801 [astro-ph.CO].

[26] W. J. Percival et al.,Mon. Not. Roy. Astron. Soc. 401 (2010) 2148, arXiv:0907.1660 [astro-ph.CO].

[27] E. A. Kazin et al., Astrophys. J. 710 (2010) 1444, arXiv:0908.2598 [astro-ph.CO].

[28] F. Beutler et al., Mon. Not. Roy. Astron. Soc. 416 (2011) 3017, arXiv:1106.3366 [astro-ph.CO].

[29] C. Blake et al., Mon. Not. Roy. Astron. Soc. 418 (2011) 1707, arXiv:1108.2635 [astro-ph.CO].

[30] N. Padmanabhan et al., Mon. Not. Roy. Astron. Soc. 427 (2012) 2132, arXiv:1202.0090 [astro-ph.CO].

[31] H.-J. Seo et al., Astrophys. J. 761 (2012) 13, arXiv:1201.2172 [astro-ph.CO].

[32] E. A. Kazin et al., Mon. Not. Roy. Astron. Soc. 441 (2014) 3524, arXiv:1401.0358 [astro-ph.CO].

[33] A. J. Ross et al., Mon. Not. Roy. Astron. Soc. 449 (2015) 835, arXiv:1409.3242 [astro-ph.CO].

[34] E. J. Copeland, M. Sami and S. Tsujikawa, Int. J. Mod. Phys. D 15 (2006) 1753, hep-th/0603057.

[35] T. Clifton, P. G. Ferreira, A. Padilla and C. Skordis, Physics Reports 513 (2012) 1, arXiv:1106.2476 
[astro-ph.CO].

[36] K. Bamba, S. Capozziello, S. Nojiri and S. D. Odintsov, Astrophys. and Space Science 342 (2012) 155, arXiv:1205.3421 [gr-qc].

[37] S. Nojiri and S. D. Odintsov, Phys. Rept. 505 (2011) 59, arXiv:1011.0544 [gr-qc].

[38] O. A. Grigorieva and G. S. Sharov, Int. J. Mod. Phys. D 22 (2013) 1350075, arXiv:1211.4992, [gr-qc].

[39] G. S. Sharov and E. G. Vorontsova, J. Cosmol. Astropart. Phys. 10 (2014) 057, arXiv:1407.5405, [gr-qc].

[40] A. Y. Kamenshchik, U. Moschella and V. Pasquier, Phys. Lett. B 511(2-4) (2001) 265, arXiv:gr-qc/0103004

[41] H. B. Benaoum, arXiv:hep-th/0205140.

[42] L. P. Chimento, Phys. Rev. D. 69 (2004) 123517.

[43] U. Debnath, A. Banerjee, S. Chakraborty, Class. Quant. Grav. 21 (2004) 5609, arXiv:gr-qc/0411015.

[44] D-J. Liu, X-Z. Li, Chin. Phys. Lett. 22 (2005) 1600, astro-ph/0501115.

[45] J. Lu, L. Xu, Y. Wu and M. Liu, Gen. Rel. Grav. 43 (2011) 819, arXiv:1105.1870 [astro-ph.CO].

[46] B. C. Paul and P. Thakur, J. Cosmol. Astropart. Phys. 11 (2013) 052, arXiv:1306.4808 [astro-ph.CO].

[47] B.C. Paul, P. Thakur and A. Beesham, arXiv:1410.6588 [astro-ph.CO].

[48] S. Nojiri and S. D. Odintsov, Phys. Rev. D 70 (2004) 103522, arXiv:hep-th/0408170

[49] S. Nojiri, S. D. Odintsov and S. Tsujikawa, Phys. Rev. D 71 (2005) 063004, arXiv:hep-th/0501025

[50] K. N. Ananda and M. Bruni, Phys. Rev. D 74 (2006) 023523, arXiv:astro-ph/0512224.

[51] E. V. Linder and R. J. Scherrer, Phys. Rev. D 80 (2009) 023008, arXiv:0811.2797 [astro-ph].

[52] A. V. Astashenok, S. Nojiri, S. D. Odintsov and A. V. Yurov, Phys. Lett. B 709 (2012) 396, arXiv:1201.4056 [gr-qc].

[53] P-H. Chavanis, arXiv:1309.5784 [astro-ph.CO].

[54] S. Nesseris and L. Perivolaropoulos, Phys. Rev. D 72 (2005) 123519, arXiv:astro-ph/0511040.

[55] O. Farooq, D. Mania and B. Ratra, Astrophys. J. 764 (2013) 139, arXiv:1211.4253 [astro-ph.CO].

[56] O. Farooq and B. Ratra, Astrophys. J. 766 (2013) L7, arXiv:1301.5243 [astro-ph.CO].

[57] K. Shi, Y. F. Huang and T. Lu, Monthly Notices Roy. Astron. Soc. 426 (2012) 2452, arXiv:1207.5875 [astro-ph.CO].

[58] A. G. Riess et al., Astrophys. J. 730(2) (2011) 119, arXiv:1103.2976 [astro-ph.CO].

[59] M. C. Bento, O. Bertolami and A. A. Sen, Phys. Rev. D 66(4) (2002) 043507, arXiv:gr-qc/0202064

[60] M. Szydłowski, A. Kurek and A. Krawiec, Phys. Lett. B 642 (2006) 171, arXiv:astro-ph/0604327. 